\title{
In the eye of the beholder
}

\author{
How do you define colour? Nina Meinzer casts light on the vision it takes.
}

ike many children, I used to annoy my mother by repeatedly asking questions I just could not answer, such as how can I explain 'red' to a blind person - or to my colour-blind grandfather? Putting aside the almost philosophical issue of relating a sensation, my childish question reduces to the problem of measuring colour - something that is more than a basic physical property.

On a first impulse many of us would probably say that colour is defined by wavelength. Yet wavelength depends on a medium's refractive index, but a blue pen will continue to look blue when immersed in water, and the same is true if the person looking at it is in water. We could obviously choose to define colour by frequency instead, which is independent of the surrounding medium's index, and this would be a natural choice for colouration produced by electronic transitions between molecular energy levels. However, the preference of frequency over wavelength is far less obvious for structurally coloured objects, for which the primarily reflected colour is dependent on the microscopic surface structure and therefore directly related to wavelength. Unlike the blue pen, the blue wings of a morpho butterfly will appear green under water.

Even if we settled on one of these standards, say wavelength, we could still not describe all colours because not every colour can be mapped onto one wavelength. Both light and pigments can be mixed to produce new colours that cannot be described by a single wavelength (or frequency) and certainly not by a simple addition or subtraction. Mixing red and yellow paint doesn't change the molecular structure of the pigments; what changes is how our brains process the wavelength information in the reflected light into our eyes. We can see that when we spin a multicoloured spinning top it appears to have only one colour while it rotates, an effect that fascinated J. C. Maxwell ${ }^{1}$.

Since colour cannot be separated from vision, we also have to take into account our perception of colour, and the most obvious way to do this is by mimicking the physiology that lets us see colours in the first place. Humans have three different types of cones - the colour receptors in the retina
- that detect different wavelength ranges. Their sensitivities are centred around a blue, a green and a red wavelength - typically around $426 \mathrm{~nm}, 530 \mathrm{~nm}$ and $555 \mathrm{~nm}$ (ref. ${ }^{2}$ ), respectively, but the exact wavelengths vary from person to person - and the cone types are often referred to as S, M and L for short, medium and long wavelength. What we see as colour is the combined response of cones, effectively mixing the colours within the response spectrum of the cones at a ratio defined by their spectral sensitivity. The most familiar example of such a trichromatic system is the RGB standard used in computer graphics, which uses experimentally determined averages of red, green and blue perception as its base colours $^{3}$ rather than the SLM wavelengths of the cones.

Unfortunately, the world of vision is not quite the same as simple RGB mixing. Cones not only respond to different wavelengths, but also have different absolute

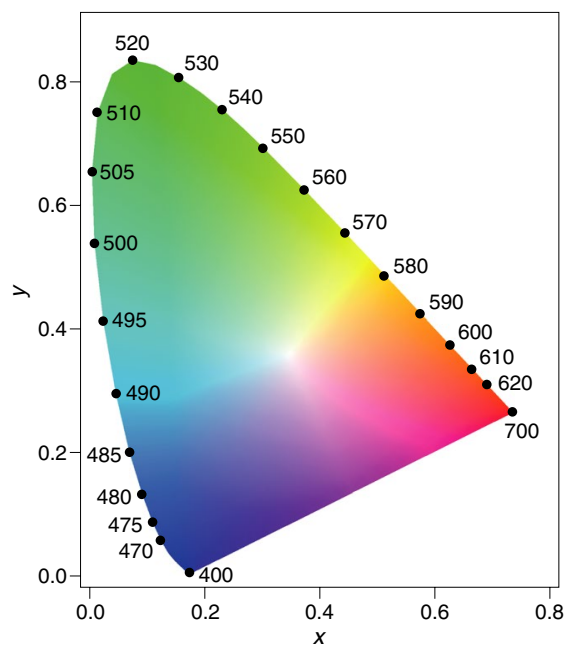

analytical expressions. These relations were first defined in 1931, along with the XYZ standard itself, by the International Commission on Illumination (CIE) after serious debate and following recommendations of researchers ${ }^{4}$; the exact definitions of the coordinates and their relations have been updated several times since. Today it is common to use the $x, y$ and $z$ coordinates, which give the proportion of the $X, Y$ and $Z$ values within their sum. No matter which coordinates one chooses, the CIE space contains all colours that an average person can see, but in the $x y$ plane the spectral colours that can be described by a single wavelength form the outline of the CIE map, as illustrated in the figure, which also shows the loci of particular monochromatic wavelengths. Because the coordinates were defined as the result of experiments on vision with several participants $^{5,6}$, they represent a standardized sensitivities. This is the reason why a green laser appears very bright at a few milliwatts while we can hardly see the output of a red titanium-sapphire laser at $1 \mathrm{~W}$. To take this into account, the widely-used CIE XYZ (1931) standard ${ }^{3}$ reduces chromatic response to two colour coordinates: $X$ corresponds approximately to the blue (S) cone and the $Z$ basis is a linear combination of the green $(M)$ and red (L) cones. This leaves the third coordinate, $Y$, to describe the luminance, which is related to the cones' sensitivities.

Unlike in the approach of mixing three primary colours and using the corresponding composition ratios to define the colour in question, the $X, Y$ and $Z$ coordinates in the CIE standard can be directly related to optical measurements (reflection, transmission and spectral radiance) using specific observer, making the CIE standard deviceinvariant. We can therefore uniquely describe the emission of, say, an LED with the colour coordinates $(0.65,0.34)$. Even my grandfather and I could have agreed on these numbers, although I still wouldn't know how he saw what I call red.

Nina Meinzer

Associate Editor, Nature Communications.

e-mail:nina.meinzer@nature.com

Published online: 2 March 2018 https://doi.org/10.1038/s41567-018-0068-1

References

1. Maxwell, J. C. Trans. R. Soc. Edinb. 21, 275-298 (1855)

2. Merbs, S. L. \& Nathans, J. Nature 356, 433-435 (1992).

3. Smith, T. \& Guild, J. Trans. Opt. Soc. 33, 73-134 (1931).

4. Troland, L. T. J. Opt. Soc. Am. 6, 527-596 (1922).

5. Wright, W. D. Trans. Opt. Soc. 30, 141-164 (1928).

6. Guild, J. Phil. Trans. R. Soc. A 230, 149-187 (1932).

$\mathrm{m} e \mathrm{~A} \mathrm{~s} R E_{\mathrm{h}}$

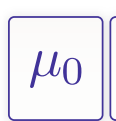

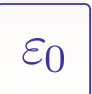

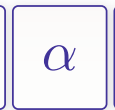

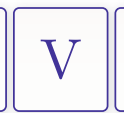

$\mathrm{R}$ 